\title{
Dabigatran-Induced Acute Interstitial Nephritis: An Important Complication of Newer Oral Anticoagulation Agents
}

\author{
Swapnil Patel ${ }^{\mathrm{a}}$, Mohammad A. Hossain ${ }^{\mathrm{a}}$, Firas Ajama ${ }^{\mathrm{a}}$, Mayurkumar Patel ${ }^{\mathrm{a}}$, \\ Mihir Nakrani ${ }^{a}$, Jasmine Patel ${ }^{\mathrm{a}}$, Alsadiq Alhillan ${ }^{\mathrm{a}}$, Mohamed Hammoda ${ }^{\mathrm{a}}$, \\ Anas Alrefaee ${ }^{\mathrm{a}}$, Michael Levitt ${ }^{\mathrm{a}}$, Arif Asif ${ }^{\mathrm{a}, \mathrm{b}}$
}

\begin{abstract}
Acute kidney injury (AKI) due to an acute interstitial nephritis (AIN) is common and can lead to increased morbidity and mortality. Medications such as antibiotics, nonsteroidal anti-inflammatory drugs (NSAIDs), proton pump inhibitors (PPI) and rifampin are common offending agents. Anticoagulant-associated AIN is more frequently reported with the use of warfarin; however, only few case reports have reported an association with the use of novel oral anticoagulants (NOACs). Herein, we report the case of a 59-year-old male who developed AKI after initiating dabigatran for the treatment of atrial fibrillation. Laboratory data demonstrated elevated blood urea nitrogen $(B U N)$ of $115 \mathrm{mg} / \mathrm{dL}$ (baseline $=35 \mathrm{mg} / \mathrm{dL}$ ) and serum creatinine $(\mathrm{Cr})$ of $5.06 \mathrm{mg} / \mathrm{dL}$ (baseline $=1.3 \mathrm{mg} / \mathrm{dL}$ ). Urinalysis revealed eosinophiluria. Renal biopsy disclosed diffuse tubulointerstitial nephritis and eosinophils and confirmed the diagnosis of AIN. At 1 week, renal function improved $(\mathrm{BUN} / \mathrm{Cr}=53 / 2.73 \mathrm{mg} / \mathrm{dL})$ with steroid therapy and discontinuation of dabigatran. With an increasing use of NOACs, it is important to monitor renal function to diagnose AIN in a timely fashion. Early diagnosis and prompt treatment can mitigate serious renal damage induced by dabigatran.
\end{abstract}

Keywords: Dabigatran; Acute interstitial nephritis; Acute kidney injury; Anticoagulation; NOAC

\section{Introduction}

Acute interstitial nephritis (AIN) is associated with interstitial inflammation of the kidney, leading to leukocyte infiltration resulting in damage and eventually an elevation of blood urea nitrogen and creatinine [1]. Antibiotics (including beta-

Manuscript submitted August 7, 2018, accepted August 21, 2018

${ }^{a}$ Department of Medicine, Jersey Shore University Medical Center, Hackensack Meridian Health, Neptune, NJ 07753, USA

${ }^{\mathrm{b}}$ Corresponding Author: Arif Asif, Department of Medicine, Jersey Shore University Medical Center, Hackensack-Meridian School of Medicine at Seton Hall, 1945 Route 33, Neptune, NJ 07753, USA.

Email: arif.asif@hackensackmeridian.org

doi: https://doi.org/10.14740/jocmr3569w lactams, sulfonamides, and rifampicin), nonsteroidal antiinflammatory drugs (NSAIDs) and proton pump inhibitors (PPI) are common agents that cause AIN [2-5]. Anticoagulant-associated AIN is more frequently reported with the use of warfarin; however, only a few case reports have been published in association with novel oral anticoagulants (NOACs) [6-10]. We report a case of a 59-year-old male who developed acute kidney injury due to AIN after initiating dabigatran for atrial fibrillation.

\section{Case Report}

A 59-year-old male, with a past medical history of paroxysmal atrial fibrillation, hypertension, diabetes mellitus, chronic kidney disease (CKD) stage 3, and osteomyelitis was referred from the outpatient clinic to the hospital for evaluation of increased blood urea nitrogen (BUN) and creatinine (Cr). Patient was receiving warfarin for atrial fibrillation, but 4 weeks prior the medication was changed to dabigatran for better compliance. A repeat of renal functions in the office showed markedly elevated serum Cr level of $4.7 \mathrm{mg} / \mathrm{dL}$ (baseline value of 1.3 $\mathrm{mg} / \mathrm{dL}$. At the time of admission the patient was asymptomatic with no evidence of hematuria, dysuria and oliguria. He had no nausea, vomiting, diarrhea, chest pain, shortness of breath, or changes in mentation. Initial laboratory investigation upon hospitalization revealed a BUN of $115 \mathrm{mg} / \mathrm{dL}$, serum creatinine of $5.06 \mathrm{mg} / \mathrm{dL}$, and eGFR of $14 \mathrm{~mL} / \mathrm{min}$ (Table 1). Patient also demonstrated serum potassium of $4.2 \mathrm{mmol} / \mathrm{L}$ and phosphorus of $6.4 \mathrm{mmol} / \mathrm{L}$. Urinalysis revealed protein of 30 $\mathrm{g} / \mathrm{L}$, large leukocytes and epithelial cells. Patient's INR was elevated at 4.01, and patient was anemic with a hemoglobin level of $9.5 \mathrm{~g} / \mathrm{dL}$ (which was around his baseline). Patient was treated with sevelamer carbonate for hyperphosphatemia. Serum ANA, ANCA and complement levels were normal. Serum protein electrophoresis (SPEP) was ordered because of proteinuria and showed mild monoclonal spike and elevated Kap$\mathrm{pa} /$ Lambda ratio. Multiple myeloma was ruled out via normal urine electrophoresis. A renal biopsy was scheduled and a urine microscopy was repeated because of a persistently elevated $\mathrm{Cr}$ level. Urine microscopy revealed numerous eosinophils. Renal biopsy was initially delayed due to hospital course being complicated by gastrointestinal bleeding secondary to arteriovenous malformation in the proximal gastric body, which was 
Table 1. Summary of Laboratory Data (Trend in BUN, Creatinine, WBC and INR)

\begin{tabular}{|c|c|c|c|c|}
\hline Laboratory examinations & Baseline & $\begin{array}{l}4 \text { weeks after initiation } \\
\text { of dabigatran }\end{array}$ & At admission & $\begin{array}{l}1 \text { week after } \\
\text { corticosteroid therapy }\end{array}$ \\
\hline \multicolumn{5}{|l|}{ Biochemistry (normal values) } \\
\hline Potassium $(3.5-5.2 \mathrm{mmol} / \mathrm{L})$ & 3.9 & 4.5 & 4.2 & 4.3 \\
\hline Chloride (96 - $110 \mathrm{mmol} / \mathrm{L})$ & 112 & 114 & 118 & 114 \\
\hline Creatinine $(0.61-1.24 \mathrm{mg} / \mathrm{dL})$ & 1.3 & 4.7 & 5.06 & 2.73 \\
\hline Glucose (70 - 99 mg/dL) & 101 & 96 & 121 & 195 \\
\hline International normalized ratio $(0.88-1.15)$ & 2.3 & 3.9 & 4.01 & 2.1 \\
\hline \multicolumn{5}{|l|}{ Complete blood count } \\
\hline Platelets $(140$ - $450 \mathrm{ku} / \mathrm{L})$ & 277 & 381 & 426 & 454 \\
\hline
\end{tabular}

Table shows the laboratory values before and 4 weeks after initiation of dabigatran, prior to hospitalization, and 1 week after initiation of corticosteroid therapy.

cauterized and stabilized. Following the kidney biopsy, the patient was empirically started on prednisone for presumed AIN. Within few days of prednisone therapy, renal function started to improve (Table 1), and repeat urine microscopy did not display any eosinophils.

Renal biopsy confirmed diffuse tubulointerstitial nephritis, acute and chronic, with a patchy increase in eosinophils and focal granulocytic tubulitis, tubular atrophy, and interstitial fibrosis (Figs. 1-3). Patient also had underlying nodular diabetic glomerulosclerosis secondary to type 2 diabetes mellitus and arteriosclerosis, with moderate arteriolosclerosis and hyalinosis.

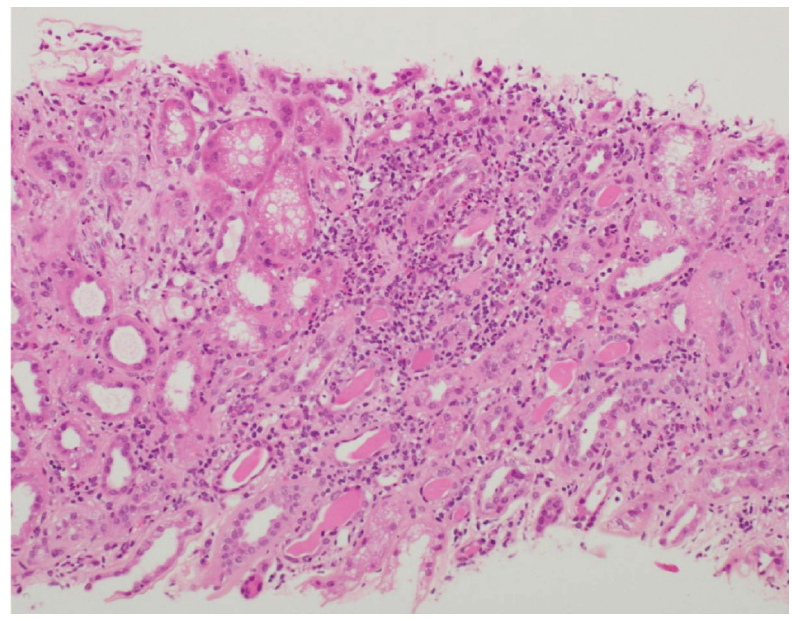

Figure 1. Light microscopy of kidney biopsy specimen (stained with $\mathrm{H} \& \mathrm{E})$ showed tubulointerstitial inflammation: interstitial inflammatory infiltrates with prominent granular sites including eosinophils and neutrophils.

\section{Discussion}

Dabigatran is a novel oral anticoagulant, which is approved by FDA for the treatment of atrial fibrillation, venous thrombosis and post-operative venous thrombosis prophylaxis [10]. Dabigatran works as a reversible direct thrombin inhibitor, preventing effects of thrombin such as activation of downstream clotting factors [11]. In our case, the patient developed acute kidney injury within 3 weeks of starting dabigatran, which improved significantly after stopping the use of dabigatran and beginning steroid therapy. The occurrence of acute kidney injury 3 weeks after the initiation of dabigatran fits in the time

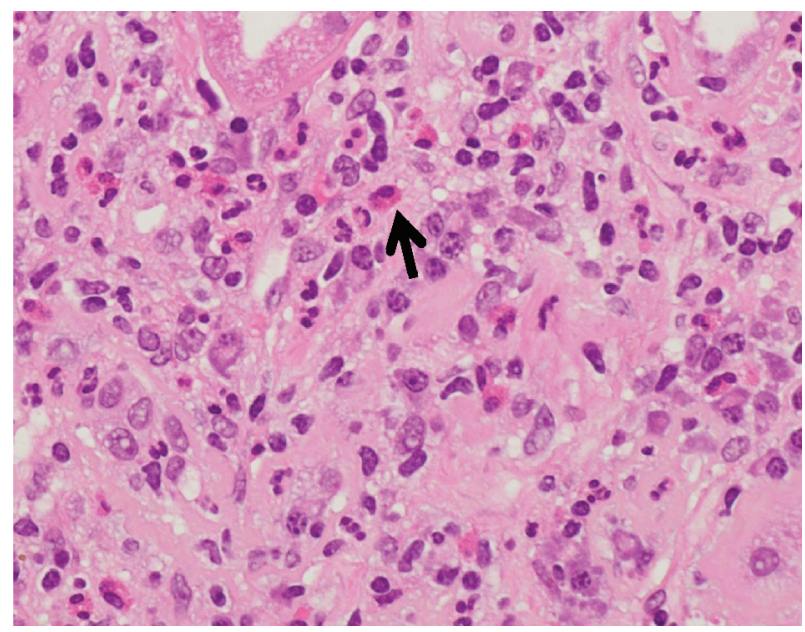

Figure 2. Light microscopy of kidney biopsy specimen stained with H\&E. Interstitial eosinophils: eosinophils (arrow), seen present within the renal interstitium diagnostic of acute interstitial nephritis 


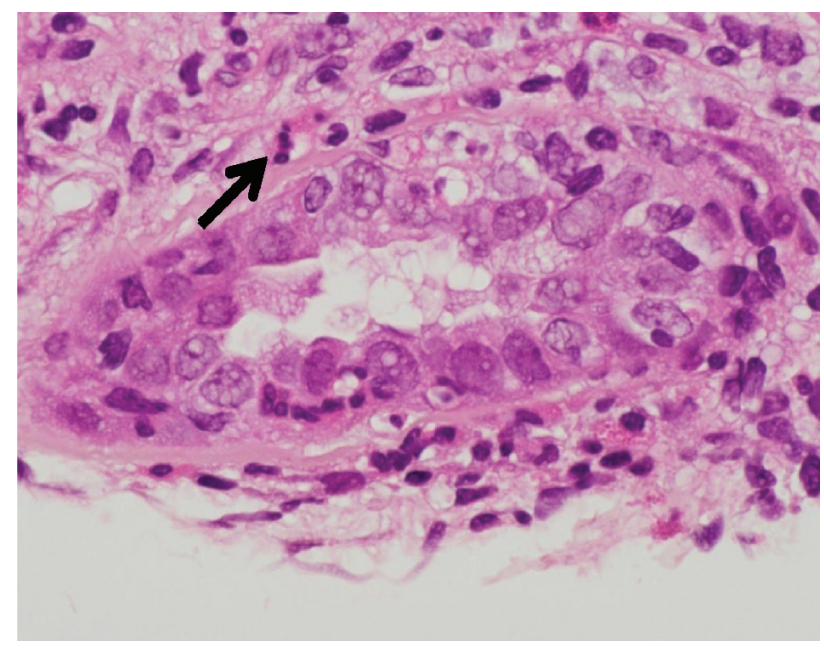

Figure 3. Granulocytic tubulitis: renal tubules seen with granulocytes including neutrophils and eosinophils (arrow).

frame for the development of AIN. Renal biopsy confirmed the diagnosis of AIN in our patient.

There have been a few case reports of acute kidney injury observed in patients being treated with dabigatran $[6,9,10$, 12]. In a case described by Escoli et al, the patient presented with acute kidney injury 2 weeks after starting dabigatran, and the patient needed to start on hemodialysis due to progressive deterioration of renal function [10]. In another case report by Kalaitzidis et al, patient developed dabigatran-induced nephropathy within a few days of starting dabigatran; however, AKI improved after stopping dabigatran only [12]. Similar to our patient, both of the patients had underlying renal disease, one had previously diagnosed glomerular disease and other had $\operatorname{IgA}$ nephropathy suggesting that pre-existing renal compromise may contribute to renal failure with anticoagulation therapy, especially dabigatran $[6,9,10,12-14]$. Renal biopsy in the reported cases revealed large intratubular red blood cell (RBC) casts with tubular necrosis and interstitial hemorrhage $[10,14]$. Immunofluorescence of a patient with underlying IgA nephropathy described by Escoli et al, showed the mesangial IgA, C3, K and Lambda chains [10]. In contrast, renal biopsy in our case showed diffuse acute tubulointerstitial nephritis, with a patchy increase in eosinophils and focal granulocytic tubulitis, tubular atrophy, and interstitial fibrosis without intratubular RBC cast and interstitial hemorrhage.

AIN is characterized by inflammation of the interstitium of the kidney, leading to edema, leukocyte infiltration, and accumulation of extracellular matrix proteins, tubular dilation and atrophy [1]. The most common cause of AIN include beta lactam antibiotics and non-steroidal anti-inflammatory drugs; however proton pump inhibitors, rifampin, systemic diseases and infections have been reported as known culprits $[2-5,15$, 16]. The exact mechanism of dabigatran-induced acute kidney injury is not clear yet. In their research, Escoli et al found that there were two proposed mechanisms of anticoagulation-associated acute kidney injury [10, 17, 18]. First, primarily being obstruction of renal tubules by RBCs, leading to vasoconstric- tion, ischemia and oxidative damage [10, 17-19]. The second mechanism involves anticoagulants decreasing thrombin activity, which leads to inhibition of protease-activated receptor 1 (PAR-1) [10, 17, 18]. Inhibition of PAR-1, which is normally a $G$ protein coupled receptor which regulates endothelial function and integrity of surrounding tissue, would lead to damage to renal tubules and interstitium and eventual decline in renal function $[10,17,18,20]$. Renal vasoconstriction inducing ischemia, promoting pro-inflammatory cytokine release and oxidative stress, all result in renal damage [12, 21, 22]. However, ours is the only case that provided direct evidence of AIN on renal biopsy induced by dabigatran (Figs. 1-3).

Management of AIN related to medications primarily consists of removing the offending agent. If there is a slow or an inadequate response, corticosteroid therapy can be considered. However, there is a lack of controlled clinical trials that support its use. However, a retrospective study conducted by Buysen et al, examined the effect of steroids on renal functions in patients with AIN [23]. Treatment group with steroids had lower serum creatinine and decreased requirements of dialysis by 18 months [23]. Our case also demonstrated benefit with steroid therapy.

\section{Conclusions}

Direct oral anticoagulants specially dabigatran, are increasingly used for better patient compliance for the treatment of venous thrombosis and atrial fibrillation. As it gains popularity, nephropathy associated with its use needs to be further researched to evaluate the pathogenesis and the identification of risk factors. However, it is of paramount importance for clinicians to monitor renal function in patients on newer oral anticoagulants therapy, especially in those with underlying CKD. Another important consideration regarding treatment modalities for AIN related to dabigatran is the role of corticosteroid therapy. Although most of these patients responded well to steroid therapy, there is a lack of controlled trials showing its benefit. It necessitates the importance of further clinical research in this field and will hopefully identify the patients at risk and a better approach to manage these groups of patients.

\section{Acknowledgments}

We would like to acknowledge the University of Columbia Medical Center for providing the images for our biopsy results.

\section{Conflict of Interest}

The authors have no conflict of interest to declare.

\section{Funding}

This project was not supported by any grant or funding agen- 
cies.

\section{Disclosure}

The patient described in the case report had given informed consent for the case report to be published.

\section{References}

1. Michel DM, Kelly CJ. Acute interstitial nephritis. J Am Soc Nephrol. 1998;9(3):506-515.

2. Schwarz A, Krause PH, Kunzendorf U, Keller F, Distler A. The outcome of acute interstitial nephritis: risk factors for the transition from acute to chronic interstitial nephritis. Clin Nephrol. 2000;54(3):179-190.

3. Brewster UC, Perazella MA. Proton pump inhibitors and the kidney: critical review. Clin Nephrol. 2007;68(2):6572.

4. Ellis T, Sunil P, Syed H, Arif A. Quiz page. A 60-year man with night sweat, and acute kidney injury. Am J Kidney Dis. 2016;63(3):xxi-xxiii.

5. Mohammad AH, Hetavi M, Attiya H, Eric JC, Hames C, Loay S, Arif A. Heorin epidemic and acute kidney injury: an under-recognized but important consequence of opiod oversode. J Med Cases. 2017;8(10):305-310.

6. Basetty P, Keith B. A cautionary tale: dabigatran toxicity associated with allergic interstitial nephritis. American Journal of Kidney Diseases. 2013;61(4):B24.

7. Abdulhadi B, Mulki R, Goyal A, Rangaswami J. Novel oral anticoagulant and kidney injury: apixaban-related acute interstitial nephritis. BMJ Case Reports. 2017.

8. Monahan RC, Suttorp MM, Gabreels B. A case of rivaroxaban-associated acute tubulointerstitial nephritis. Neth J Med. 2017;75(4):169-171.

9. Kadiyala D, Brewster UC, Moeckel GW. Dabigatran induced acute kidney injury. Proceedings of the American Society of Nephrology Annual Meeting. 2012.

10. Escoli Rachele, et al. Dabigatran-related nephropathy in a patient with undiagnosed IgA nephropathy. Case Reports in Nephrology. 2015;2015:1-4.

11. Ganetsky M, Babu KM, Salhanick SD, Brown RS, Boyer EW. Dabigatran: review of pharmacology and management of bleeding complications of this novel oral anticoagulant. J Med Toxicol. 2011;7(4):281-287.

12. Kalaitzidis RG, Duni A, Liapis G, Balafa O, Xiromeriti S, Rapsomanikis PK, Elisaf MS. Anticoagulant-related nephropathy: a case report and review of the literature of an increasingly recognized entity. Int Urol Nephrol. 2017;49(8):1401-1407.

13. Brodsky SV, Nadasdy T, Rovin BH, Satoskar AA, Nadasdy GM, Wu HM, Bhatt UY, et al. Warfarin-related nephropathy occurs in patients with and without chronic kidney disease and is associated with an increased mortality rate. Kidney Int. 2011;80(2):181-189.

14. Brodsky SV, Satoskar A, Chen J, Nadasdy G, Eagen JW, Hamirani M, Hebert L, et al. Acute kidney injury during warfarin therapy associated with obstructive tubular red blood cell casts: a report of 9 cases. Am J Kidney Dis. 2009;54(6):1121-1126.

15. Kodner CM, Kudrimoti A. Diagnosis and management of acute interstitial nephritis. Am Fam Physician. 2003;67(12):2527-2534.

16. Muriithi AK, Leung N, Valeri AM, Cornell LD, Sethi S, Fidler ME, Nasr SH. Biopsy-proven acute interstitial nephritis, 1993-2011: a case series. Am J Kidney Dis. 2014;64(4):558-566.

17. Ware K, Qamri Z, Ozcan A, Satoskar AA, Nadasdy G, Rovin $\mathrm{BH}$, Hebert LA, et al. N-acetylcysteine ameliorates acute kidney injury but not glomerular hemorrhage in an animal model of warfarin-related nephropathy. Am J Physiol Renal Physiol. 2013;304(12):F1421-1427.

18. Martin Cleary C, Moreno JA, Fernandez B, Ortiz A, Parra EG, Gracia C, Blanco-Colio LM, et al. Glomerular haematuria, renal interstitial haemorrhage and acute kidney injury. Nephrol Dial Transplant. 2010;25(12):4103-4106.

19. Rizk DV, Warnock DG. Warfarin-related nephropathy: another newly recognized complication of an old drug. Kidney Int. 2011;80(2):131-133.

20. Tracz MJ, Alam J, Nath KA. Physiology and pathophysiology of heme: implications for kidney disease. J Am Soc Nephrol. 2007;18(2):414-420.

21. Moreno JA, Martin-Cleary C, Gutierrez E, Rubio-Navarro A, Ortiz A, Praga M, Egido J. Haematuria: the forgotten CKD factor? Nephrol Dial Transplant. 2012;27(1):2834.

22. Gutierrez E, Egido J, Rubio-Navarro A, Buendia I, Blanco Colio LM, Toldos O, Manzarbeitia F, et al. Oxidative stress, macrophage infiltration and CD163 expression are determinants of long-term renal outcome in macrohematuria-induced acute kidney injury of IgA nephropathy. Nephron Clin Pract. 2012;121(1-2):c42-53.

23. Buysen JG, Houthoff HJ, Krediet RT, Arisz L. Acute interstitial nephritis: a clinical and morphological study in 27 patients. Nephrol Dial Transplant. 1990;5(2):94-99. 